Conclusions Pazopanib may be better tolerated than sunitinib, with an acceptable adverse event profile and fewer dose adjustments.

Also, the severity of adverse events looks lower with pazopanib.

However, the number of patients was too small to arrive at definitive conclusions, so it is necessary to enlarge this study.

No conflict of interest.

\section{GRP-169 SCREENING FOR CLINICALLY RELEVANT INTERACTIONS IN LIVER TRANSPLANT PATIENTS}

doi:10.1136/ejhpharm-2013-000276.169

${ }^{1} \mathrm{C}$ Andrés González, ${ }^{2} \mathrm{RM}$ Romero Jimenez, ${ }^{3} \mathrm{E}$ Valverde Alcalá, ${ }^{3}$ I Muñoz Castillo. ${ }^{1} H R U$ CARLOS HAYA, pharmacy, Malaga, Spain; 'HRU GREGORIO MARAÑÓN, pharmacy, Madrid, Spain; ${ }^{3}$ HRU CARLOS HAYA, pharmacy, Madrid, Spain

Background Drug-drug interactions are a frequent problem in liver transplant (LT) patients, further hindering pharmacotherapeutic management, which is a very important risk to the patient's life. Purpose To detect drug-drug interaction of clinical relevance in LT patients in a tertiary hospital.

Materials and Methods Descriptive transversal study of the LT patients in our hospital during 2011 who were admitted to the Digestive Surgery Unit (DSU). Variables analysed were: sex, number of drugs prescribed at admission and number of days of hospitalisation in the DSU. Data were collected from clinical and pharmacotherapeutic histories and the unit dose dispensing log. Drug-drug interactions were detected and analysed by the Micromedex Healthcare series ${ }^{\circledR}$ database. The results were analysed with the SPSS v.19 statistics software.

Results Of a total of 51 transplant patients, we included 44 (5 patients died and in 2 patients the medicines were not recorded at admission to the DSU).

$75 \%$ of patients were male and $25 \%$ female, mean age of patients was $53 \pm 12$ years. The median number of days in hospital was 11 [9.18] days. The mean number of drugs prescribed on admission was $11 \pm 2.5 \mathrm{drugs} /$ patient

The total number of drug interactions detected was 210 of which $153(72.9 \%)$ were clinically relevant, representing a prevalence of $84.1 \%$ of liver transplant patients.

Of the main variables studied, only the number of drugs prescribed was found to be directly proportional $(p<0.05)$ to the number of clinically relevant interactions detected, thus no relationship was obtained between age or the number of days hospitalised.

Conclusions Liver transplant patients are critically ill patients with highly complex treatment. A high prevalence of clinically relevant interactions was detected related to polypharmacy and the use of high-risk medicines.

The presence of a pharmacist in this Unit would be beneficial to comprehensively review these patients' treatment.

No conflict of interest.

\section{GRP-170 SEARCHING FOR THE CAUSE OF ALLERGIC CUTANEOUS ADVERSE DRUG REACTIONS: RETROSPECTIVE ANALYSIS OF A FIVE-YEAR CLINICAL EXPLORATION IN A SINGLE- CENTRE COHORT}

doi:10.1136/ejhpharm-2013-000276.170

${ }^{1} \underline{G}$ Maton, ${ }^{2} \mathrm{C}$ Brzezinki, ${ }^{1} \mathrm{H}$ Beaussart, 'D Lannoy, ${ }^{2} \mathrm{D}$ Staumont-Sallé, ${ }^{1} \mathrm{P}$ Odou. ${ }^{1}$ Centre Hospitalier Regional Universitaire, Pharmacy, Lille, France, ${ }^{2}$ Centre Hospitalier Regional Universitaire, Dermatology, Lille, France

Background Adverse drug reactions on skin affect approximately $2 \%$ of patients. Skin and drug challenge tests were performed in the dermatology department to assess these reactions and pharmacy-compounded drugs were tested through patches, pricks and intradermal (IDR) tests.

Purpose To assess the incidence of positive allergic reactions in tested patients and to define the culprit drugs and their potential allergic role in these reactions.

Materials and Methods The study was conducted between 2007 and 2010 on patients from our hospital. We collected information on the characteristics of the adverse drug reaction on skin, the drugs tested, the tests performed and their results.

Results In the period studied, 220 patients referred by other practitioners (from the hospital or from ambulatory practitioners) for serious cutaneous reactions were tested and 3225 preparations were performed by the pharmacy. 92 patients had an immediate reaction to the drug and 128 had a non-immediate reaction. $64(29 \%)$ patients developed a positive response: $48(75 \%)$ through skin tests (patch, prick and IDR) and 16 (25\%) through a Drug Challenge Test (DCT). The drugs most often involved in the positive tests were anti-infectious drugs (46\%), paracetamol (16\%) and iodinated contrast media (10\%).

Conclusions The percentage of positive tests in this cohort agrees with the data found in the literature (3-76\%). The large difference is due to the variability in patient recruitment.

However, it is difficult to compare these data because the preparation and interpretation of the tests are not standardised.

Allergology tests still improve the care of patients as with negative skin tests and DPTs many patients were able to continue with their treatment.

Manufacturing tests by the pharmacy standardise preparation conditions within the hospital and reduce cross contamination and microbial contamination.

No conflict of interest.

\section{GRP-171 SECURING INTRATHECAL INJECTIONS: WHAT ABOUT NON-LUER CONNECTORS?}

doi:10.1136/ejhpharm-2013-000276.171

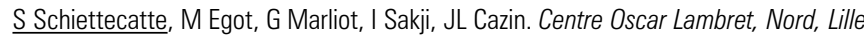
Cedex, France

Background Episodes of accidental injection of medicines intended for intravenous administration into the intrathecal space have been reported worldwide, often leading to death. Since 2001, international guidelines have been issued to prevent such risks. A major recommendation is to develop a non-luer connector to use in neuraxial procedures

Purpose To give an overview of the development and marketing of medical devices fitted with non-luer connectors.

Materials and Methods Manufacturers' catalogues have been consulted. A literature review was conducted using the PubMed and Science Direct databases, including the following MeSH keywords 'non luer', 'connectors', 'safety' and 'intrathecal'. European Health Authorities websites have been also consulted. All searches were performed between August and October 2012.

Results The United Kingdom, which has been a pioneer in guidance, was the first to implement such connectors. Five different non-luer connectors have been designed thanks to the National Patient Safety Agency (NPSA) initiative. Literature research identified few individual tests of these new devices. Some incidents such as mismatching connectors have been documented. So the NPSA has updated recommendations about introducing secure non-luer connectors. These devices are coming onto the French and Belgian market soon. To our knowledge safety connectors are not yet available in other countries.

Conclusions Non-luer connectors for intrathecal drug administration were initially launched in Great Britain. This process obviously 
improves the safety of intrathecal injections and leads other countries in the same way. However more advanced scientific studies of these connectors should be published. The main line of thought should be the standardisation of these connectors. Lack of standardisation is generating some hazards and supervised implementation of these medical devices is required.

No conflict of interest.

\section{GRP-172 SELECTION AND IMPLEMENTATION OF PERFORMANCE INDICATORS MEASURING THE QUALITY OF THE CLINICAL PHARMACY SERVICE OF THE MATER MISERICORDIAE UNIVERSITY HOSPITAL}

doi:10.1136/ejhpharm-2013-000276.172

'B Ryan, 'P Ging, 'J Brown, ${ }^{2} \mathrm{R}$ Edwards, 'C Meegan. 'Mater Misericordiae University Hospital, Pharmacy Department, Dublin 7, Ireland (Rep.); 'Robert Gordon University, Pharmacy Department, Aberdeen, UK

Background The Health Information and Quality Authority (HIOA) in Ireland are currently promoting and guiding the development of key performance indicators and minimum data sets to monitor health care quality. A third of Irish hospital pharmacies surveyed in 2006 believed that performance indicators were the most effective quality assessment tool. Despite this, performance indicators for clinical pharmacy services in Ireland have not been published.

Purpose To obtain consensus on whether performance indicators identified from the literature provide a valid and feasible method of measuring the quality of the Mater Misericordiae University Hospital (MMUH) clinical pharmacy service and whether they could be introduced as a regular quality measurement.

Materials and Methods Review the literature relating to the use of performance indicators in a clinical pharmacy setting and identify performance indicators which have been piloted or used in other institutions.

Achieve consensus of a multidisciplinary panel, using a Delphi method of the most valid and feasible performance indicators for the MMUH clinical pharmacy service

Implement one of the selected performance indicators

Make recommendations on the further use of performance indicators

Results Performance indictors relating to hospital pharmacy are available $(\mathrm{n}=240)$ in the literature.

The Delphi method achieved consensus and rated the following three performance indicators as both valid and feasible:

Percentage of reserve antimicrobials checked by a clinical pharmacy for approval by microbiology or infectious diseases

Percentage of patients discharged on warfarin who receive warfarin counselling by a clinical pharmacist

Percentage of medication orders for intermittent therapy that have been reviewed by a clinical pharmacist for safe prescribing.

The indicator chosen for measurement was the percentage of medication orders for intermittent therapy that were reviewed by a clinical pharmacist for safe prescribing. A $79 \%$ compliance with this performance indicator was achieved by the clinical pharmacy service.

Conclusions A multidisciplinary panel achieved consensus that three of the performance indicators identified from the literature provide valid and feasible methods of measuring the quality of the clinical pharmacy service of the MMUH. One of these was successfully implemented and consideration will be given to implementing further performance indicators

No conflict of interest.

\section{GRP-173 SEVERE ANAEMIA CAUSED BY DRUG INTERACTION. A CASE STUDY}

doi:10.1136/ejhpharm-2013-000276.173

M Perpinya, L Mallart, D Malla, E De Puig. Institut d'Assitència Sanitària, Pharmacy, Salt (Girona), Spain

Background Retrospective study based on the clinical history and the Naranjo causality algorithm.

Purpose To describe a case of severe anaemia in a HIV-positive patient receiving zidovudine and lamotrigine

Materials and Methods A 54-year old male HIV patient on antiretroviral therapy since 2002 (zidovudine $300 \mathrm{mg} / 12 \mathrm{~h}$, lamivudine $150 \mathrm{mg} / 12 \mathrm{~h}$ and abacavir $300 \mathrm{mg} / 12 \mathrm{~h}$ ), with partial epileptic seizures treated with lamotrigine (100 mg/12 h) since May 2011 who in 2007 developed low haemoglobin and haematocrit levels. A diagnosis of macrocytic anaemia was made and the patient was followed up every six months without treatment. In July 2011, at the Drug Care Unit, very low levels of haemoglobin (RBCs $1.17 \mathrm{M} / \mathrm{mcL}$, haemoglobin $5 \mathrm{~g} / \mathrm{dL}$, haematocrit 15\% and MCV $128 \mathrm{fL}$ ), asthenia, weight loss, and dyspnoea upon exertion were detected. These findings were reported to the treating doctor and the patient was admitted, with temporary discontinuation of antiretroviral and antiepileptic treatment. While in hospital, the patient required three consecutive erythrocyte concentrate transfusions.

Results At 8 weeks post-transfusion and discontinuation of antiretroviral and antiepileptic therapy, the patient's blood levels returned to normal. Antiretroviral and antiepileptic therapy was reinitiated with different drugs.

The causality relationship between severe macrocytic anaemia and zidovudine was shown to be 'probable' using the Naranjo Algorithm. Zidovudine causes macrocytic anaemia described in the data sheet as 'frequent' (1\%). According to the lamotrigine data sheet, haematological alterations are rare $(<0.01 \%)$. In this case, the macrocytic anaemia that was probably caused by zidovudine might have been made worse by a drug that rarely presents haematological toxicity.

Conclusions Macrocytic anaemia is a common serious adverse reaction to zidovudine. This drug can also cause accumulated toxicity when administrated with drugs that may also cause haematological alterations. Patients receiving these drugs require close monitoring and coordination between physician and pharmacist.

No conflict of interest.

\section{GRP-174 SIGNIFICANCE OF POTENTIALLY INAPPROPRIATE MEDICINES FOR ELDERLY PATIENTS AT A GERMAN UNIVERSITY HOSPITAL}

doi:10.1136/ejhpharm-2013-000276.174

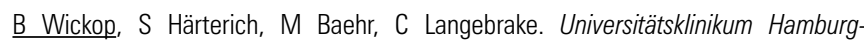
Eppendorf, Pharmacy, Hamburg, Germany

Background Certain drugs are classified as potentially inappropriate medication (PIM) for the elderly because they bear an increased risk of adverse drug events resulting in major safety concerns. Several classifications have been published to identify and avoid PIM. For this study FORTA [1] (fit for the aged), PRISCUS [2] (Latin: time-honoured) and STOPP [3] (Screening Tool of Older Persons' potentially inappropriate Prescriptions) criteria have been chosen as the most relevant ones.

Purpose The aims are to determine which PIM are taken by elderly patients at University Medical Center Hamburg-Eppendorf (UKE) and how the prevalence of PIM changes from admission to discharge

Materials and Methods Based on the criteria provided by FORTA, PRISCUS and STOPP, medication of patients $>65$ years is 